\title{
Normoglycemic Diabetic Nephropathy: The Role of Insulin Resistance
}

\author{
Edward J. Filippone ${ }^{a} \quad$ Astha Gupta $^{a} \quad$ John L. Farber ${ }^{b}$ \\ ${ }^{a}$ Division of Nephrology, Department of Medicine and ${ }^{b}$ Department of Pathology, \\ Thomas Jefferson University Hospital, Philadelphia, Pa., USA
}

\section{Key Words}

Diabetic nephropathy · Insulin resistance $\cdot$ Idiopathic nodular glomerulosclerosis · Metabolic syndrome

\begin{abstract}
The pathophysiology of diabetic nephropathy (DN) is complex and incompletely understood. Whereas hyperglycemia is clearly important, the role of insulin resistance (IR) is increasingly recognized. We present the case of a normotensive non-smoking obese woman with nephrotic syndrome who was found to have DN by biopsy. All measures of glucose metabolism, including fasting glucose, glycosylated hemoglobin, and oral glucose tolerance testing, were repeatedly normal with little exception. IR was documented, however, based on the presence of the metabolic syndrome and an elevated homeostasis model assessment of IR. We posit that this IR is central to the pathogenesis of our patient's lesion, and this may explain other cases of DN with normoglycemia. The literature supporting this concept is discussed.

(C) 2014 S. Karger AG, Basel
\end{abstract}

\section{Introduction}

Diabetic nephropathy (DN) is the most common cause of end-stage renal disease (ESRD), accounting for nearly half of the incident cases in the United States [1]. The nephropathies of types 1 and 2 diabetes mellitus (DM) are identical [2]. The clinical manifestations are also similar with early glomerular hyperfiltration, followed by progressively increasing proteinuria, hypertension, and eventual loss of glomerular filtration rate (GFR). 
Approximately $25-50 \%$ of patients with types 1 or 2 DM eventually develop clinical manifestations of DN, but the pathophysiology of DN remains incompletely understood. Both observational data and randomized controlled trials of intensive therapy demonstrate the central role of hyperglycemia and its attendant metabolic consequences in the initiation and progression of DN [3, 4]. Of possibly similar importance is insulin resistance (IR), especially in light of recent data demonstrating the insulin responsiveness of renal epithelial cells [5].

We present the case of a young woman who first developed proteinuria as a pregnant teenager and who eventually became persistently nephrotic. Biopsy revealed DN, although hyperglycemia was never evident. She did have IR, however, and we argue that this was the main factor in the pathogenesis of her DN.

\section{Case Report}

A 27-year-old African American female was referred for nephrotic syndrome in 2012. In 2005, during her first pregnancy, she had developed proteinuria and preeclampsia. The proteinuria subsided after she had given birth; however, it recurred during a second pregnancy in 2006 and increased to the nephrotic range. The second pregnancy was otherwise uneventful, and after delivery, the degree of proteinuria trended down to a nadir of $120 \mathrm{mg} / \mathrm{g}$ creatinine by 2009 . She subsequently presented to our hospital in 2012 when the proteinuria again increased, reaching $4,600 \mathrm{mg} / 24 \mathrm{~h}$ (or 2,900 mg/g creatinine).

The patient had no past medical history of diabetes, hypertension, or other systemic illnesses. She was not taking any medications, had no known allergies and denied ever smoking or having used illicit drugs. Of note, both her father and maternal grandmother had type $2 \mathrm{DM}$ and her father had had a stroke at age 50.

On examination, her blood pressure was 100/60 mm Hg and her BMI was 33. There was no edema, acanthosis nigricans, or hirsuitism. Fundoscopy did not reveal any diabetic retinopathy. Laboratory values included $0.7 \mathrm{mg} / \mathrm{dl}$ serum creatinine, $167 \mathrm{mg} / \mathrm{dl}$ total cholesterol, $42 \mathrm{mg} / \mathrm{dl} \mathrm{HDL}$ cholesterol, and $77 \mathrm{mg} / \mathrm{dl}$ triglycerides. Urinalysis showed 3+ proteinuria with no hematuria. Tests for HIV, RPR, HBV, HCV, ANA, C3, and C4 were all negative or normal. Fasting glucose was $80 \mathrm{mg} / \mathrm{dl}$ and $\mathrm{Hb}_{\mathrm{A} 1 \mathrm{C}}$ was $5.8 \%$. Renal ultrasound revealed kidneys $12 \mathrm{~cm}$ in size.

A renal biopsy was obtained in October 2012. Of the 7 glomeruli present, none were globally or partially sclerotic. Glomerulomegaly was not present. Using an eyepiece micrometer, the mean glomerular diameter was $175 \pm 12 \mu \mathrm{m}$, a value within the reported normal range. One glomerulus evidenced segmental hyalinosis. Otherwise, the glomeruli were unremarkable by light microscopy (fig. 1a). Evidence of chronicity such as interstitial fibrosis and tubular atrophy was absent. Immunofluorescence microscopy revealed mild (1$2+$ ) pseudolinear staining of the glomerular basement membrane (GBM) for IgG. No other immunoglobulin classes or complement components were detectable. Electron microscopy showed that the lamina densa of the GBMs were diffusely thickened, averaging 700-900 nm (fig. 1b). The podocytes were not vacuolated and, importantly, their foot processes were not effaced (fig. 1b). The endothelial cells were swollen with loss of fenestrations. Hyaline deposits were also noted in the glomerular capillary wall (fig. 1c), a finding corresponding to the light microscopic changes mentioned above.

Our patient was serially evaluated for impaired glucose metabolism. Fasting blood glucoses ranged from 80 to $111 \mathrm{mg} / \mathrm{dl}$ (with only two values $\geq 100 \mathrm{mg} / \mathrm{dl}$ ). $\mathrm{Hb}_{\mathrm{A} 1 \mathrm{C}}$ ranged from 5.6 to 5.9\%. Fluorescein angiography was negative for diabetic retinopathy. An oral glucose tolerance test (OGTT) was normal with a 2-hour glucose level of $109 \mathrm{mg} / \mathrm{dl}$. The 
homeostasis model assessment of IR (HOMA-IR) was elevated at 2.93 and 3.74, a result that indicates IR (normal value: 1 ) in the face of adequate insulin secretion to maintain normoglycemia and a normal OGTT.

\section{Discussion}

Our patient had evidence of pathologic DN features: diffuse GBM thickening, arteriolar hyalinosis, and pseudolinear IgG GBM staining. This would be considered Class IIa in the recent Renal Pathology Society classification of DN [2], although the criteria to diagnose DM were not met.

The pathophysiology of DN remains incompletely understood. Multiple factors are implicated, including genetic predisposition, metabolic derangements (hyperglycemia, nonenzymatic glycosylation products, hyperinsulinemia/IR, etc.), hemodynamic factors, inflammation, and the complex interplay of numerous hormones, cytokines, and growth factors. We discuss the central role IR may play in the development of diabetic glomerulosclerosis, as was the case in our patient [5].

The gold standard method for the determination of IR involves the whole body glucose disposal rate as determined by a hyperinsulinemic-euglycemic clamp; specifically, the rate of glucose infusion required to maintain normoglycemia in the face of a constant insulin infusion. Alternatively, less invasive methods include the quantitative insulin sensitivity check index (QUICKI) and the HOMA-IR, both using fasting glucose and insulin levels [6]. The HOMA-IR (HOMA-IR = fasting glucose $\times$ fasting insulin/22.5) is a simplified predictor of IR when only fasting levels of glucose and insulin are available, and it correlates with IR more than insulin sensitivity. An individual with 'normal' insulin sensitivity will have a value of 1 , and there is a reasonable linear correlation between the HOMA-IR and hyperinsulinemiceuglycemic clamp [7]. Our patient had elevated HOMA-IR levels both times it was determined. However, HOMA-IR is not an equivalent measure of IR to the hyperinsulinemiceuglycemic clamp, because it is restricted to the fasting state [8]. An OGTT can provide similar information to the HOMA-IR if concurrent insulin and glucose levels are obtained, with the additional evidence of dynamic function after a glucose load [8]. Insulin levels were not drawn during the OGTT of our patient.

A strong and reciprocal association exists between chronic kidney disease (CKD) and IR. IR is well known to exist with uremia, but it can also be demonstrated at even early stages of CKD. Furthermore, studies in the general population show a significant relationship between IR and the development of CKD [9].

Most patients with either type 1 or 2 DM will not develop overt nephropathy. However, evidence supports a role for the IR degree as one determining factor in the minority of the type 1 patients that do develop DN [10-14]. Type 2 DM is clearly an insulin-resistant state. Nevertheless, patients with type 2 DM who develop DN have been shown to be more insulin resistant than those who do not. For example, Parvanova et al. [15] compared 50 microalbuminuric to 50 normoalbuminuric type 2 diabetic patients, and found the glucose disposal to be $25 \%$ lower in the former [15]. No difference was evident, however, when comparing 29 microalbuminuric to 29 macroalbuminuric patients, confirming that IR is present early in the course of the disease. Hsu et al. [16] prospectively followed 738 normoalbuminuric type 2 diabetic patients for five years and found that the development of microalbuminuria was significantly higher in the 3rd and 4th highest quartiles of the baseline HOMA-IR than in the lowest quartile [16]. 
Of obvious importance are the mechanisms by which IR can lead to DN: either indirectly through hyperglycemia, hyperinsulinemia, hyperlipidemia, etc., or directly due to renal IR per se. Both prior and current research has centered on the mesangium. More recently, the critical role of the podocyte in DN has become apparent, recognizing normal insulin signaling as being necessary for proper functioning.

Abnormalities of the structure and number of podocytes can be demonstrated in both types of DM [17-19]. Verzola et al. [20] demonstrated podocyte apoptosis in biopsies of patients with early type 2 DN. Podocyte apoptosis was not further increased with more advanced nephropathy and was not found in other glomerulopathies. In a study of 41 Pima Indians, Pagtalunan et al. [21] found a reduced podocyte number per glomerulus in microalbuminuric and macroalbuminuric patients as compared to normoalbuminuric patients with a similar duration of DM. Meyer et al. [22] biopsied 16 microalbuminuric diabetic Pima Indians and then followed them for four years. Ten became macroalbuminuric, and the strongest predictor of renal disease progression was the baseline podocyte number per glomerulus. Similar findings were reported by Dalla Vestra et al. [23] in 67 Italian patients. Podocyte density (not number) per glomerulus differentiated the proteinuric patients from the normoalbuminuric diabetic controls. Such podocyte abnormalities may be directly attributable to IR.

Podocytes express insulin receptors and require normal insulin signaling for sustained viability. In vitro, podocytes respond to insulin at physiologic levels by translocating the glucose transporters GLUT1 and GLUT4 to the cell membrane, with a resultant doubling of glucose uptake [24]. This response is dependent on both a normally functioning actin cytoskeleton [24] and nephrin [25]. Reduced nephrin expression occurs in both early and advanced DN, and is required for normal podocyte insulin signaling.

Impaired insulin signaling in various experimental models of DN is reported both in vivo and in vitro. Insulin receptor activation results in the phosphorylation of protein kinase B (Akt), which is a key survival protein [26]. In a study by Tejada et al [26], phosphorylated Akt was lower in vivo in the glomeruli of the insulin-resistant $\mathrm{db} / \mathrm{db}$ mouse, a model of type 2 DM. Podocytes in vitro from $\mathrm{db} / \mathrm{db}$ mice had reduced pAKT and increased apoptosis [26]. In a model most germane to our case, Welsh et al. [27] generated mice with a specific deletion of the insulin receptor in podocytes alone. These animals had normal extra-renal insulin sensitivity but podocyte-specific IR. Despite persisting normoglycemia, they developed albuminuria by 5 weeks. Subsequently, progressive structural damage appeared, including podocyte apoptosis, foot process fusion, a thickened glomerular basement membrane, and glomeruloscerosis. However, it is unlikely that IR alone is sufficient in humans, given its wide prevalence and the apparent rarity of cases such as ours.

As in our patient, pathological evidence of DN in the absence of overt DM has occurred before. Some of the reported patients had impaired glucose metabolism that was below the threshold to diagnose DM, and they would be classified as having impaired fasting glucose or impaired glucose tolerance. Others had glucose levels diagnostic of DM either previously, concurrent, or subsequent to their renal biopsy.

Nevertheless, patients with all measures of glucose metabolism within the normal range or close to it have been reported. In 1999, Herzenberg et al. [28] described two such cases and coined the term idiopathic nodular glomerulosclerosis (ING). In 2002, Markowitz et al. [29] reported the largest series of ING (23 patients) and noted that most of the patients were elderly (mean age 68), hypertensive (95\%), hyperlipidemic (90\%), and frequently used tobacco (91\%). Eleven had some evidence, however, of abnormal glucose metabolism. The authors postulated that the combination of long-standing hypertension and smoking could result in the development of this ING [29]. Kuppachi et al. [30] reviewed 46 cases of ING said 
to have normal glucose metabolism (including the 23 of the study by Markowitz et al.) and found that $82 \%$ had hypertension and $88 \%$ smoked. Smoking itself may result in the production of reactive glycosylation products, termed glycotoxins, which are capable of forming advanced glycosylation end products in vitro and in vivo [31]. Smoking has been shown to increase the risk for microalbuminuria in patients with type 1 DM [32].

Clear evidence of IR, however, was present in many of the reported cases of ING, and may be of prime importance in its pathophysiology. Many showed frankly abnormal glucose metabolism [30, 33, 34], and others had evidence of MS, especially obesity [35]. Nevertheless, DN is pathologically distinct from obesity-related glomerulopathy, a lesion that is primarily characterized by glomerulomegaly and features of focal segmental glomerulosclerosis. In the largest series of obesity-related glomerulopathy, $45 \%$ had some changes consistent with DN (both GBM thickening and mesangial sclerosis) [36]. Although GBM thickening was present, it was only mild and segmental. By contrast, our patient had neither glomerulomegaly (mean diameter $175 \mu \mathrm{m}$, within the normal range [36]) nor focal segmental scars, but there was prominent diffuse GBM thickening (700-900 nm). Thus, she did not have obesity-related glomerulopathy or any other definable glomerulopathy. Other entities mimicking DN by light microscopy include amyloidosis, fibrillary/immunotactoid glomerulonephritis, monoclonal immunoglobulin deposition diseases, and fibronectin glomerulopathy. These have been ruled out by the serologic and ultrastructural findings in our case.

In conclusion, our patient illustrates the possible role of IR in the development of DN in the absence of glucose intolerance. We suspect that many similar patients who may be labeled as having normoglycemic DN (or ING if nodules are present) may have underlying IR and need to be formally tested. While IR is not sufficient to cause DN, it may interact with other factors (genetic or hemodynamic factors, smoking, etc.) to produce such a lesion in the absence of overt hyperglycemia.

\section{Disclosure Statement}

The authors have no conflicts of interest to declare.

\section{References}

1 De Boer IH, Rue TC, Hall YN, et al: Temporal trends in the prevalence of diabetic kidney disease in the United States. JAMA 2011;305:2532-2539.

-2 Tervaert TWC, Mooyart AL, Amann K, et al: Pathological classification of diabetic nephropathy. J Am Soc Nephrol 2010;21:556-563.

-3 The Diabetes Control and Complications Trial Research Group: The effects of intensive treatment of diabetes on the development and progression of long term complications in insulin dependent diabetes mellitus. N Engl J Med 1993;329:977-986.

-4 UK Prospective Diabetes Study (UKPDS) Group: Intensive blood glucose control with sulphonylureas or insulin compared with conventional treatment and risk of complications in patients with type 2 Diabetes. Lancet 1998;352:837-853.

5 De Cosmo S, Menzaghi C, Prudente S, Trischitta V: Role of insulin resistance in kidney dysfunction: insights into the mechanism and epidemiological evidence. Nephrol Dial Transplant 2013;28:29-36.

6 Mathews DR, Hosker JP, Rudenski AS, et al: Homeostasis model assessment: insulin resistance and $\beta$-cell function from fasting plasma glucose and insulin concentrations in man. Diabetologia 1985;28:412-419.

-7 Muniyappa R, Lee S, Chen H, Quon M: Current approaches for assessing insulin sensitivity and resistance in vivo: advantages, limitations, and appropriate usage. Am J Physiol Endocrinol Metab 2008;294:E15-E26.

-8 Pacini G, Mari A: Methods for clinical assessment of insulin sensitivity and beta-cell function. Best Pract Res Clin Endocrinol Metab 2003;17:305-322. 


\begin{tabular}{l|l}
\hline Case Rep Nephrol Urol 2014;4:137-143 \\
\hline DOI: 10.1159/000364901 & $\begin{array}{l}\text { @ 2014 S. Karger AG, Basel } \\
\text { www.karger.com/cru }\end{array}$ \\
\hline
\end{tabular}

Filippone et al.: Normoglycemic Diabetic Nephropathy: The Role of Insulin Resistance

9 Chen J, Munter P, Lee Hamm L, et al: Insulin resistance and risk of chronic kidney disease in nondiabetic US adults. J Am Soc Nephrol 2003;14:469-477.

10 Chaturvedi N, Bandinelli S, Mangili R, et al: Microalbuminuria in type 1 diabetes: rates, risk factors and glycemic threshold. Kidney Int 2001;60:219-227.

11 De Boer IH, Sibley SD, Kestenbaum B, et al: Central obesity, incident microalbuminuria, and change in creatinine in the epidemiology of diabetes interventions and complications study. J Am Soc Nephrol 2007;18:235-243.

12 Yip J, Mattock MB, Morocutti A, et al: Insulin resistance in insulin-dependent diabetic patients with microalbuminuria. Lancet 1993;342:883-887.

$\$ 13$ Ekstrand AV, Groop PH, Grönhagen-Riska C: Insulin resistance precedes microalbuminuria in patients with insulin-dependent diabetes mellitus. Nephrol Dial Transplant 1998;13:3079-3083.

14 Orchard TJ, Chang YF, Ferrell RE, et al: Nephropathy in type 1 diabetes: a manifestation of insulin resistance and multiple genetic susceptibilities? Kidney Int 2002;62:963-970.

15 Parvanova AI, Trevisan R, Iliev IP, et al: Insulin resistance and microalbuminuria. A cross-sectional, casecontrol study of 158 patients with type 2 diabetes and different degress of urinary albumin excretion. Diabetes 2006;55:1456-1462.

16 Hsu CC, Chang HY, Huang MC, et al: Association between insulin resistance and development of microalbuminaria in type 2 diabetes. Diabetes Care 2011;34:982-987.

17 Ellis EN, Steffes MW, Chavers B: Observation of glomerular epithelial cell structures in patients with type 1 diabetes mellitus. Kidney Int 1987;32:736-741.

18 Toyoda M, Najafian B, Kim Y, et al: Podocyte detachment and reduced glomerular capillary endothelial fenestration in human type 1 diabetic nephrpathy. Diabetes 2007;56:2155-2160.

19 White KE, Bilous RW, Marshall SM, et al: Podocyte number in normotensive type 1 diabetic patients with albuminuria. Diabetes 2002;51:3083-3089.

20 Verzola D, Gandolfo MT, Rastaldi MP, et al: Apoptosis in the kidneys of patients with type 2 diabetic nephropathy. Kidney Int 2007;72;1262-1272.

21 Pagtalunan ME, Miller PL, Jumping-Eagle S, et al: Podocyte loss and progressive glomerular injury in type 2 diabetes. J Clin Invest 1997;99:342-348.

-22 Meyer TW, Bennett PH, Nelson RG: Podocyte number predicts long-term urinary albumin excretion in Pima Indians with type 2 diabetes and microalbuminuria. Diabetologia 1999;42:1341-1344.

-23 Dalla Vestra M, Masiero A, Roiter AM, et al: Is podocyte injury relevant in diabetic nephropathy. Studies in patients with type 2 diabetes. Diabetes 2003;52:1031-1035.

24 Coward RJM, Welsh GL, Yang J, et al: The human glomerular podocyte is a novel target for insulin action. Diabetes 2005;54:3095-3102.

25 Coward RJM, Welsh GL, Koziell A, et al: Nephrin is critical for the action of insulin on human glomerular podocyte. Diabetes 2007;56:1127-1135.

-26 Tejada T, Catanuto P, Ijaz A, et al: Failure to phosphorylate AKT in podocyte from mice with early diabetic nephropathy promotes cell death. Kidney Int 2008;73:1385-1393.

27 Welsh G, Hale L, Eremina V, et al: Insulin signaling to glomerular podocyte is critical for normal kidney function. Cell Metab 2010;12:329-340.

-28 Herzenberg AM, Holden JK, Singh S, et al: Idiopathic nodular glomerulosclerosis. Am J Kidney Dis 1999;34:560-564.s

29 Markowitz GS, Lin J, D'Agati et al: Idiopathic nodular glomerulosclerosis is a distinct clinicopathologic entity linked to hypertension and smoking. Hum Pathol 2002;33:825-835.

-30 Kuppachi S, Idris N, Chander PN, Yoo J: Idiopathic nodular glomerulosclerosis in a non-diabetic hypertensive smoker - case report and review of literature. Nephrol Dial Transplant 2006;21:3571-3575.

31 Cerami C, Founds H, Nicholl I, et al: Tobacco smoke is a source of toxic reactive glycation products. Proc Natl Acad Sci 1997;94:13915-13920.

32 Chase PH, Garg SK, Marshall G, et al: Cigarette smoking increases the risk of albuminuria among subjects with type 1 diabetes. JAMA 1991;265:614-617.

-33 Sanai T, Okuda S, Yoshimitsu T, et al: Nodular glomerulosclerosis in patients without any manifestation of diabetes mellitus. Nephrology 2007;12:69-73.

34 Mac-Moune Lai F, Szeto CC, Choi PC, et al: Isolate diffuse thickening of glomerular capillary basement membrane: a renal lesion in prediabetes? Mod Path 2004;17:1506-1512.

-35 Li W, Verani RR: Idiopathic nodular glomerulosclerosis: a clinicopathologic study of 15 cases. Hum Path 2008;39:1771-1776.

-36 Kambham N, Markowitz G, Valeri A: Obesity related glomerulopathy: an emerging epidemic. Kidney Int 2001;59:1498-1509. 


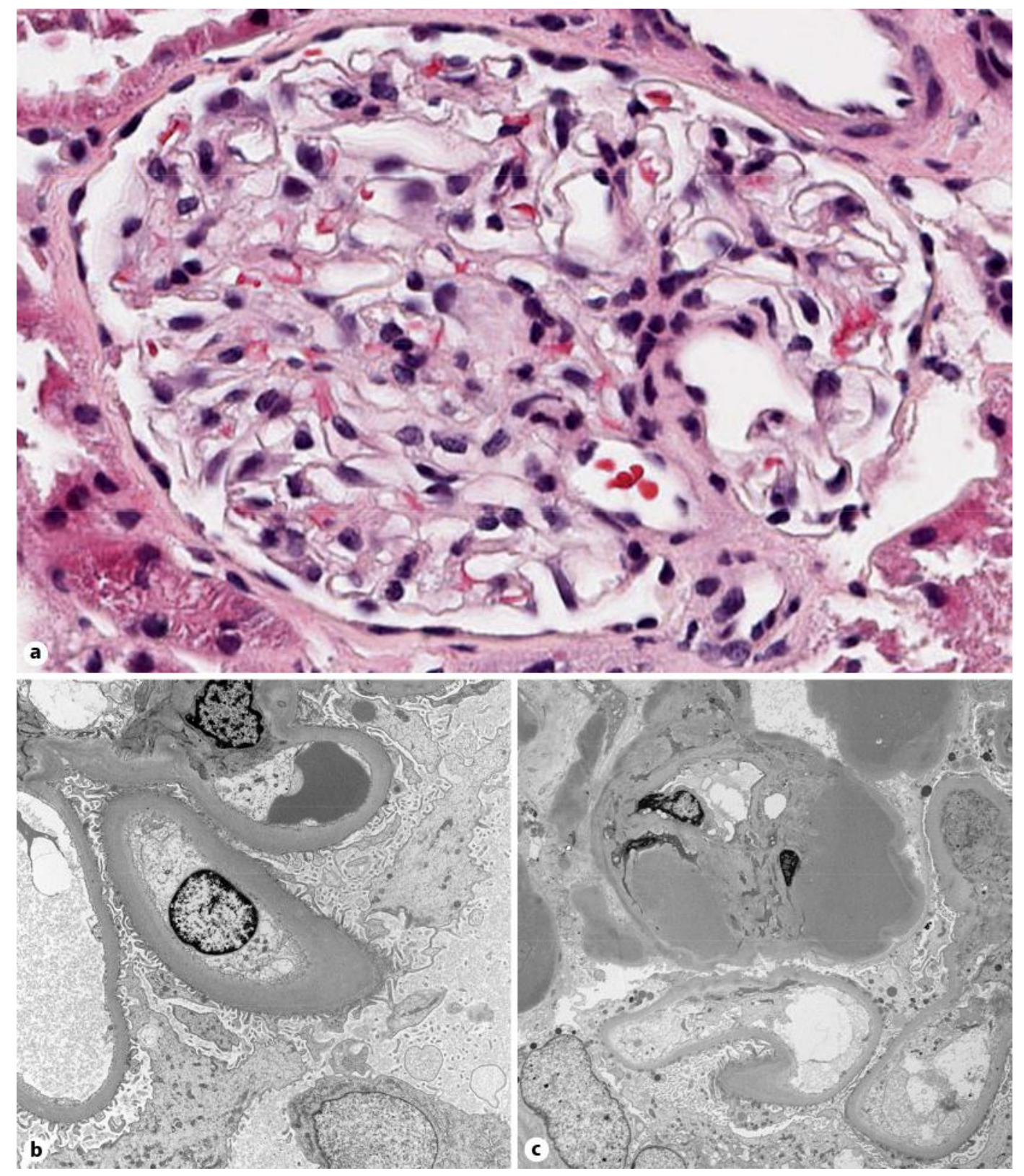

Fig. 1. a Light microscopy. Photomicrograph of a representative glomerulus showing normal histology (HE. $\times 40)$. b Electron microscopy. Diffuse thickening of the lamina densa of the glomerular basement membranes is evident. The epithelial cells are unremarkable, whereas the endothelial swells are swollen with loss of fenestrations $(\times 4,000)$. c Electron microscopy. Prominent subendothelial hyaline deposits $(\times 2,700)$. 